\title{
Hydrogen bonding and the design of twist-bend nematogens
}

Rebecca Walker1*, Damian Pociecha2, Catriona Crawford1, John MD Storey1, Ewa Gorecka2 and Corrie T Imrie 1

1Department of Chemistry, King's College, University of Aberdeen, Aberdeen, AB24 3UE, UK.*E-mail: rebecca.walker@abdn.ac.uk

2Department of Chemistry, University of Warsaw, ul. Zwirki i Wigury 101, 02-089 Warsaw, Poland.

\begin{abstract}
The transitional properties of equimolar mixtures consisting of a hydrogen bond donor, a 4alkoxybenzoic acid ( $n \mathrm{OBA})$, and one of two different stilbazole-based hydrogen bond acceptors, either 4-[(E)-2-(4-\{[6-(4'-methoxy[1,1'-biphenyl]-4-yl)hexyl]oxy $\}$ phenyl)ethenyl]-pyridine (1OB6OS) or 4-[(E)-4'-(6-\{4-[(E)-2-(pyridin-4-yl)ethenyl]phenoxy $\}$ hexyl)[1,1'-biphenyl]-4-carbonitrile (CB6OS) are reported. Neither hydrogen bond acceptor exhibits liquid crystal behaviour whereas the $n \mathrm{OBA}$ series shows smectic and / or nematic behaviour depending on the length of the alkyloxy chain. For the mixtures containing either hydrogen bond acceptor and an $n \mathrm{OBA}$ with $\mathrm{n}=1-5$, both conventional nematic and twist-bend nematic phases were observed. On increasing $n$ smectic phases emerged and the twist-bend nematic phase was extinguished. The CB6OS- $n$ OBA mixtures may exhibit the heliconical smectic $\mathrm{C}_{\mathrm{TB}}$ phase. The local molecular arrangement in the two sets of mixtures are similar and changes on increasing $n$ but this is not reflected in the nematic-isotropic transition temperatures. Birefringence studies of the mixtures are reported. In general the behaviour of the hydrogenbonded mixtures is similar to that of their covalently bonded counterparts,
\end{abstract}

\section{Introduction}

The spontaneous emergence of chirality in systems composed of achiral molecules is of fundamental importance in both physical and biological sciences and thought to play a pivotal role in the origin of biological homochirality. In this context the study of liquid crystalline systems has greatly enhanced our understanding of symmetry breaking in fluids 1 . Indeed, the first example of spontaneous chiral symmetry breaking in a fluid with no spatial ordering was provided by the twist-bend nematic, $\mathrm{N}$ TB, phase in which the director maps out a conical helix 
and is tilted at a constant angle with respect to the helical axis 2. In this system consisting of achiral molecules, chirality forms spontaneously and so an equal number of left- and righthanded helices would be expected. The pitch of the helix is strikingly small, of the order of 10 $\mathrm{nm}$, which corresponds to just a few molecular lengths. 3. The introduction of intrinsic or molecular chirality removes the degeneracy of the helices and the chiral NTB phase is observed 4. Prior to its experimental discovery, the $\mathrm{N}_{\text {тв }}$ phase had been predicted first by Meyer 5 and later and independently by Dozov 6. In these models the director modulations arose from flexoelectric couplings, or from an elastic instability equivalent to a sign change of the bend elastic constant (K33), respectively. Within Dozov's framework, bent molecules are required to obtain the necessary low values of $\mathrm{K}_{33}$ and the vast majority of twist-bend nematogens are oddmembered liquid crystal dimers 7-14. These consist of molecules containing two mesogenic units attached through a flexible alkyl spacer, and if the parity of the spacer is odd then the molecule, on average, is bent 15 . The NTB phase is most often preceded by a conventional nematic phase with uniform director structure but having an anomalously low bend elastic constant, and such materials can be exploited in new applications 16,17. However, direct NTBisotropic transitions have been observed 18,19. In addition, Dozov predicted that bent achiral molecules would form chiral twist-bend smectic phases 6 and these have also been observed experimentally $11,20,21$.

The Nтв phase has been found for a range of other structures including bent-core liquid crystals 22, and higher liquid crystal oligomers such as trimers and tetramers 22-24. In each of these systems molecular curvature is thought to be the essential structural requirement for the formation of the NTB phase. Hydrogen bonding has also been employed in the design of twistbend nematogens, and the first example was 4-[6-(4'-cyanobiphenyl-4-yl)hexyloxy]-benzoic acid (CB6OBA), see Figure 1. $\{\mathrm{REF}\}$ The observation of Nтв behaviour was attributed to the formation of hydrogen-bonded complexes between pairs of acids yielding bent supramolecular complexes 26. In this system, however, there is no element of molecular recognition given that two identical molecules form the complexes. In order to extend this design approach and build in an aspect of molecular recognition, we reported recently the behaviour of mixtures containing unlike hydrogen bond acceptors and donors, namely 4-[(E)-2-(4-\{[6-(4'methoxy[1,1'-biphenyl]-4-yl)hexyl]oxy\}phenyl)ethenyl]-pyridine (1OB6OS) and alkoxybenzoic acids ( $n \mathrm{OBA}$ ) with $n=4$ and 5 27, respectively, see Table 1, and showed that these systems do indeed exhibit the Nтв phase providing examples of the formation of spontaneous chirality driven by hydrogen bonding between unlike and achiral components. To 
further investigate this fascinating behaviour, here we report the behaviour of mixtures containing a wider range of the hydrogen bond donors $n \mathrm{OBA}$ in which the length of the terminal chain has been varied, $n=1-10$ and 1OB6OS, and also mixtures containing a different stilbazole-based hydrogen bond acceptor, 4-[(E)-4'-(6-\{4-[(E)-2-(pyridin-4yl)ethenyl]phenoxy\}hexyl)[1,1'-biphenyl]-4-carbonitrile (CB6OS), see Table 1. These particular systems have been chosen for study because the hydrogen bond acceptors both contain a hexyloxy spacer which has been shown to impart the required molecule curvature for the observation of twist-bend behaviour 7 and both the methoxy and cyano terminal groups have been shown to promote the Nтв phase 28-30. Furthermore, the hydrogen bond between pyridyl-based fragments and benzoic acids has been shown to be sufficiently strong to promote liquid crystalline complexes 31-33. By analogy with covalently assembled systems 10,11, the extension of the terminal chain associated with the hydrogen bond donor may drive the formation of smectic as well as nematic phases.

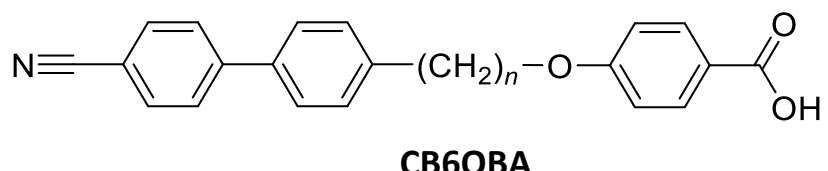



Table 1. Hydrogen bond acceptors and hydrogen bond donors used in this study.

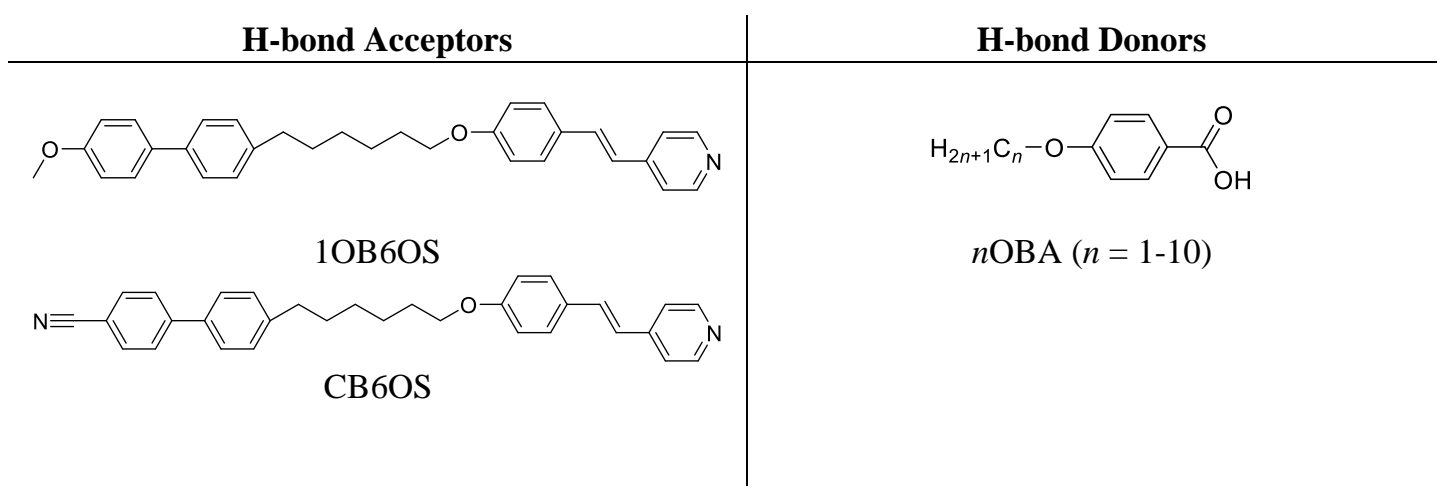

\section{Experimental}

\section{Synthesis}

nOBAs 
The synthesis of the $n$-alkoxybenzoic acids ( $n$ OBAs) followed a standard procedure adapted from that reported by Jensen et al. 34 and is shown in Scheme 1.<smiles>[R]Oc1ccc(C(=O)O)cc1</smiles>

Scheme 1. Synthesis of the $n$-alkoxybenzoic acid ( $n \mathrm{OBA})$ series, where $\mathrm{R}=\mathrm{C}_{\mathrm{n}} \mathrm{H}_{2 \mathrm{n}+1}(n=1-10)$.

Full synthetic details and analytical data for $n$ OBA with $n=1-10$ are given in the ESI.

\section{B60S}

The synthesis of 4-[(E)-2-(4-\{[6-(4'-methoxy[1,1'-biphenyl]-4-yl)hexyl]oxy\}phenyl)-ethenyl]pyridine (1OB6OS) followed the procedure outlined in Scheme 2. A Friedel-Crafts acylation and subsequent ketone reduction gave 1OB6Br. The alcoholic stilbazole 4-[(E)-2-(pyridin-4-yl)ethenyl]phenol (HOS) was prepared through the base-catalysed condensation of 4-hydroxybenzaldehyde and 4methylpyridine. 35 1OB6Br and HOS were combined in a Williamson ether synthesis to give the title compound.

Full synthetic details and analytical data for 1OB6OS and its intermediates are given in the ESI.

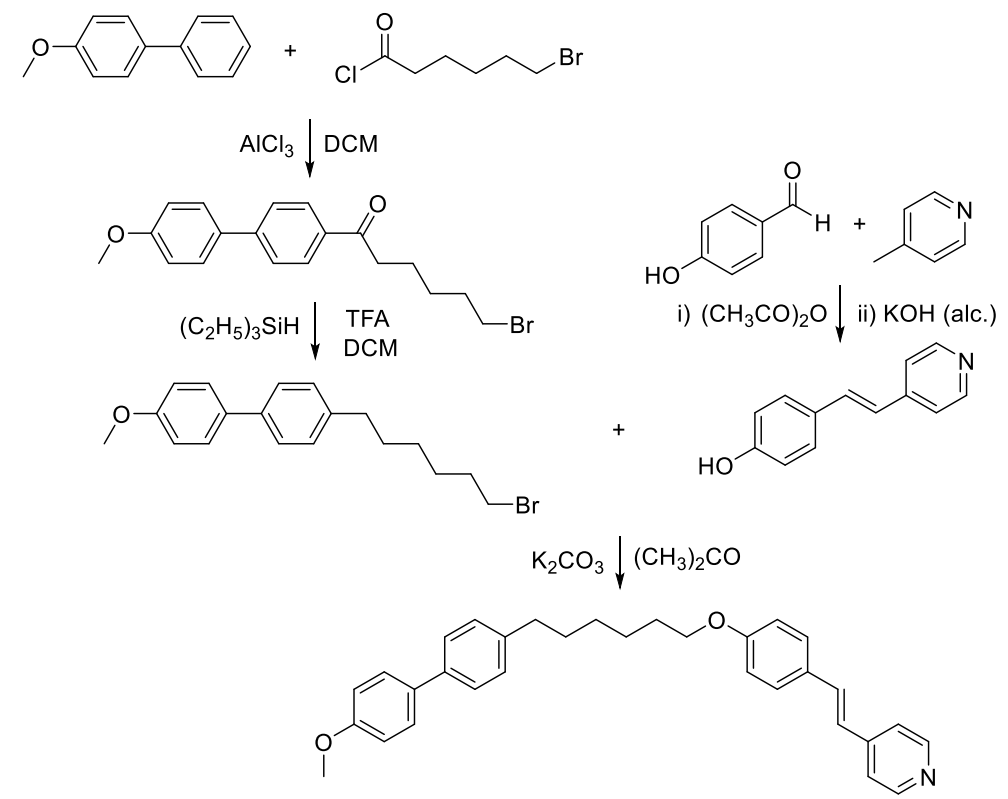

Scheme 2. Synthesis of 1OB6OS.

\section{CB6OS}


The synthesis of CB6OS followed the procedure outlined in Scheme 3. The synthesis of 4'-[6-(4formylphenoxy)hexyl][1,1'-biphenyl]-4-carbonitrile, $\mathrm{CB} 6 \mathrm{OAH}$, and its intermediates was performed as described in an earlier work.11 The condensation of CB6OAH and 4-methylpyridine gave the title compound. Full synthetic details and analytical data for CB6OS and its intermediates are given in the ESI.

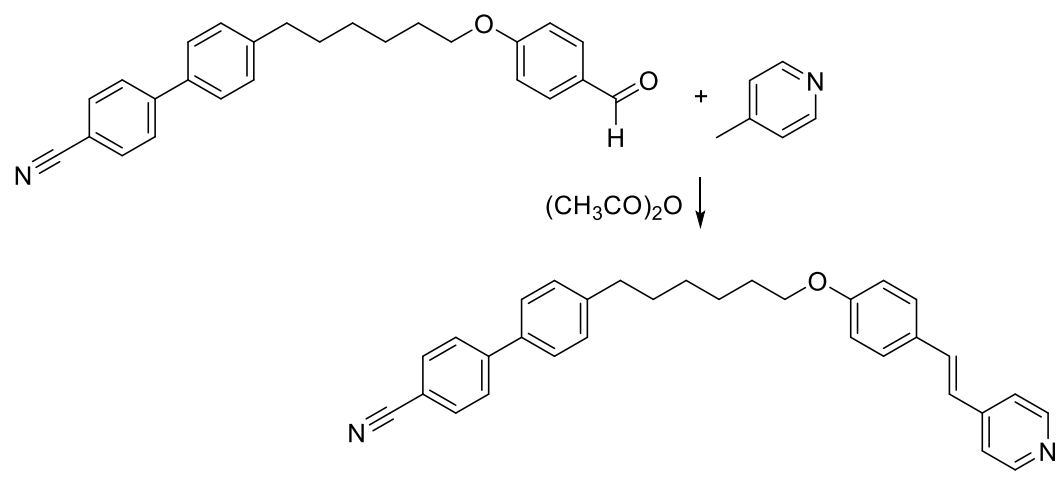

Scheme 3. Synthesis of CB6OS.

\section{Materials and Methods}

Materials All reagents and solvents available commercially were purchased from Sigma Aldrich, Alfa Aesar, ACROS Organics or TCI Chemicals and used as received unless stated otherwise.

Purity Analysis The proposed structures of all final products and their intermediates were characterised using a combination of ${ }_{1} \mathrm{H}$ and ${ }_{13} \mathrm{C}$ NMR, and FTIR spectroscopies. ${ }_{1} \mathrm{H}$ and ${ }_{13} \mathrm{C}$ NMR spectra were recorded on either a $400 \mathrm{MHz}$ Varian Unity INOVA, or a $300 \mathrm{MHz}$ Bruker Ultrashield NMR spectrometer. Infrared spectra were recorded on a Thermal Scientific Nicolet IR100 FTIR spectrometer with an ATR diamond cell. The purities of the final products were verified using $\mathrm{C}, \mathrm{H}, \mathrm{N}$ microanalysis performed by the Micro Analytical Laboratory in the School of Chemistry at the University of Manchester or Sheffield Analytical and Scientific Services Elemental Microanalysis Service at the University of Sheffield.

Polarised Optical Microscopy Phase characterisation was performed using an Olympus $\mathrm{BH} 2$ polarising light microscope equipped with a Linkam TMS 92 hot stage (University of Aberdeen) or a Zeiss AxioImager A2m polarizing microscope equipped with Linkam heating stage (University of Warsaw). Glass cells were obtained from Warsaw Military University of Technology (WAT) having 1.6 or 3 micron thicknesses, and ITO conducting and 60 polymer aligning layers were used.

Differential Scanning Calorimetry The phase behaviour of the dimers was studied by differential scanning calorimetry using a Mettler-Toledo DSC1 fitted with an intracooler and calibrated using 
indium and zinc as standards. Heating and cooling rates were $10^{\circ} \mathrm{C}$ min-1 and all samples were measured under a nitrogen atmosphere. Transition temperatures and associated enthalpy changes were extracted from the second heating trace unless otherwise noted. For each sample, two aliquots were measured and the data listed are the average of the two sets of data.

X-ray Diffraction Wide angle diffractograms (WAXS) were obtained with a Bruker D8 GADDS system $(\mathrm{CuK} \alpha$ line, Goebel mirror, point beam collimator, Vantec2000 area detector). Samples were prepared as droplets on a heated surface.

Birefringence Birefringence was measured with a set-up based on a photoelastic modulator (PEM-90, Hinds) working at a modulation frequency $\mathrm{f}=50 \mathrm{kHz}$; as a light source, a halogen lamp (Hamamatsu LC8) was used equipped with a narrow bandpass filters (633 nm and $690 \mathrm{~nm}$ ). The signal from a photodiode (FLC Electronics PIN-20) was deconvoluted with a lock-in amplifier (EG\&G 7265) into 1f and $2 \mathrm{f}$ components to yield a retardation induced by the sample. Knowing the sample thickness, the retardation was recalculated into optical birefringence. Prior to analysis, the samples were placed in glass cells provided by Warsaw Military University of Technology (WAT) having 3 micron thickness, and ITO conducting and 60 polymer aligning layers. The alignment quality was checked prior to measurement by inspection under the polarised optical microscope.

Binary Mixture Studies Binary mixtures were prepared by co-dissolving pre-weighed amounts of each compound in dichloromethane or chloroform and allowing the solvent to evaporate slowly at room temperature. The mixtures were further dried in a vacuum oven at $50{ }^{\circ} \mathrm{C}$ for $\sim 16 \mathrm{~h}$.

\section{Results and Discussion}

The transitional properties of the equimolar 1OB6OS: $n$ OBA complexes are listed in Table 2, and those of the individual components are given in Tables S1-3 in the ESI. 1OB6OS melts into the isotropic phase at $143{ }^{\circ} \mathrm{C}$, and on cooling crystallisation precluded any observable liquid crystallinity. All ten complexes show a conventional enantiotropic nematic, $\mathrm{N}$, phase assigned on the basis of the observation of a characteristic schlieren texture containing two- and fourpoint brush singularities in untreated cells, and a uniform texture in cells with planar anchoring, see Figure 2. For the 1OB6OS: $n$ OBA complexes with $n=1-5$, a transition to a lower temperature, monotropic nematic phase was apparent by the development of a striped texture, characteristic of the Nтв phase, see Figure 2. The N-NTв phase transition was accompanied by the cessation of optical flickering associated with director fluctuations in the higher temperature $\mathrm{N}$ phase. For the 1OB6OS:5OBA complex, the temperature range of the Nтв phase narrows to only ca. $4 \mathrm{~K}$, insufficient for the striped texture to develop prior to a transition to a 
smectic A, SmA, phase. The N-Nтв transition was detected using DSC as a very small jump in the heat capacity, in accord with the general observation that as the temperature range of the preceding nematic phase increases, the N-NTв transition tends towards becoming second order in nature, i.e. $\Delta \mathrm{H} \approx 030$.

In the equimolar 1OB6OS:nOBA complexes with $n>5$, the Nтв phase is no longer observed and instead the nematic phase cools directly into a SmA phase. The SmA phase is assigned on the basis of the observation of a truncated focal conic fan texture, indicative of smectic layer ordering, in coexistence with homeotropic regions indicating that the director is orthogonal to the layer planes. The entropy change associated with the SmA-N transition increases as the temperature range of the $\mathrm{N}$ phase decreases in accord with McMillan theory and the values observed are consistent with the phase assignment.36 In thin cells sample crystallisation is suppressed, and cooling from either the Nтв phase $(n=3-4)$ or the SmA phase $(n=5-10)$ revealed a reversible transition to another smectic phase, $\mathrm{SmX}$, having a mosaic-like texture, see Figure 2. The monotropic nature of the SmX phase precluded its study using X-ray diffraction but given the large enthalpy change associated with its formation, it is presumably a highly ordered smectic phase.

Table 2. Transition temperatures and associated entropy changes for the 1OB6OS: $n$ OBA equimolar mixtures. All data extracted from DSC heating traces unless stated otherwise. *Temperature obtained from DSC cooling trace. $\dagger$ Temperature obtained using POM. $\ddagger$ Peak overlapped with crystallisation exotherm.

\begin{tabular}{|c|c|c|c|c|c|c|c|c|c|c|}
\hline$n$ & $\begin{array}{l}\mathrm{TCr}- \\
/{ }^{\circ} \mathrm{C}\end{array}$ & $\begin{array}{c}\mathbf{T}_{\text {SmASmX }} \\
/^{\circ} \mathrm{C}\end{array}$ & $\begin{array}{c}\text { TSmAN }_{\text {Sm }} \\
/{ }^{\circ} \mathrm{C}\end{array}$ & $\begin{array}{c}\mathbf{T}_{\mathbf{N B}_{\mathbf{T B}}}{ } \\
/{ }^{\circ} \mathbf{C}\end{array}$ & $\begin{array}{r}\mathbf{T}_{\mathrm{NI}} \\
/{ }^{\circ} \mathbf{C}\end{array}$ & $\begin{array}{c}\Delta \mathrm{SCr}_{\mathrm{C}} / \\
\mathbf{R}\end{array}$ & $\begin{array}{c}\Delta \operatorname{SSmASmX}_{\mathrm{S}} / \\
\mathbf{R}\end{array}$ & $\begin{array}{c}\Delta \mathrm{SSmAN}_{\mathrm{N}} / \\
\mathbf{R}\end{array}$ & $\begin{array}{c}\Delta \mathbf{S}_{\mathbf{T}_{\mathbf{T B}}} / \\
\mathbf{R}\end{array}$ & $\begin{array}{c}\Delta \mathbf{S}_{\mathrm{NI}} / \\
\mathbf{R}\end{array}$ \\
\hline 1 & 132 & & & $104 \dagger$ & 173 & 15.59 & & & $\approx 0$ & 0.70 \\
\hline 2 & 120 & & & $98 \dagger$ & 168 & 10.94 & & & $\approx 0$ & 0.66 \\
\hline 3 & 115 & $77 *$ & & $99 \dagger$ & 164 & 10.94 & $t$ & & $\approx 0$ & 0.63 \\
\hline 4 & 122 & $86^{*}$ & & $102 \dagger$ & 166 & 14.22 & 2.42 & & $\approx 0$ & 0.65 \\
\hline 5 & 112 & $85^{*}$ & $94 *$ & $98 \dagger$ & 158 & 12.26 & 1.41 & 0.08 & $\approx 0$ & 0.57 \\
\hline 6 & 119 & $86^{*}$ & $102 *$ & & 155 & 8.54 & 1.40 & 0.08 & & 0.59 \\
\hline 7 & 97 & $89 *$ & 114 & & 152 & 10.31 & 1.10 & 0.04 & & 0.73 \\
\hline 8 & 101 & $96 *$ & 127 & & 155 & 8.82 & 1.61 & 0.11 & & 0.65 \\
\hline 9 & 95 & $92 *$ & 127 & & 149 & 5.82 & 1.32 & 0.15 & & 0.71 \\
\hline 10 & 106 & $99 *$ & 138 & & 153 & 9.71 & 2.17 & 0.21 & & 0.71 \\
\hline
\end{tabular}


Figure 2. POM textures of the 1OB6OS:3OBA equimolar mixture in a cell with planar anchoring in the $\mathrm{N}$ and $\mathrm{N}$ тв phases, and for 1OB6OS:5OBA in the SmA and SmX phases.

The phase behaviour of the equimolar 1OB6OS:nOBA complexes was studied further using $\mathrm{X}$-ray diffraction. The $\mathrm{N}$ and $\mathrm{N}$ тв phases show essentially identical X-ray patterns with broad signals indicative of the liquid-like arrangement of the molecules. Table 3 lists the periodicities measured along the director in the liquid crystal phases of these mixtures along with the estimated molecular lengths of the complexes. The very weak reflections seen in the X-ray
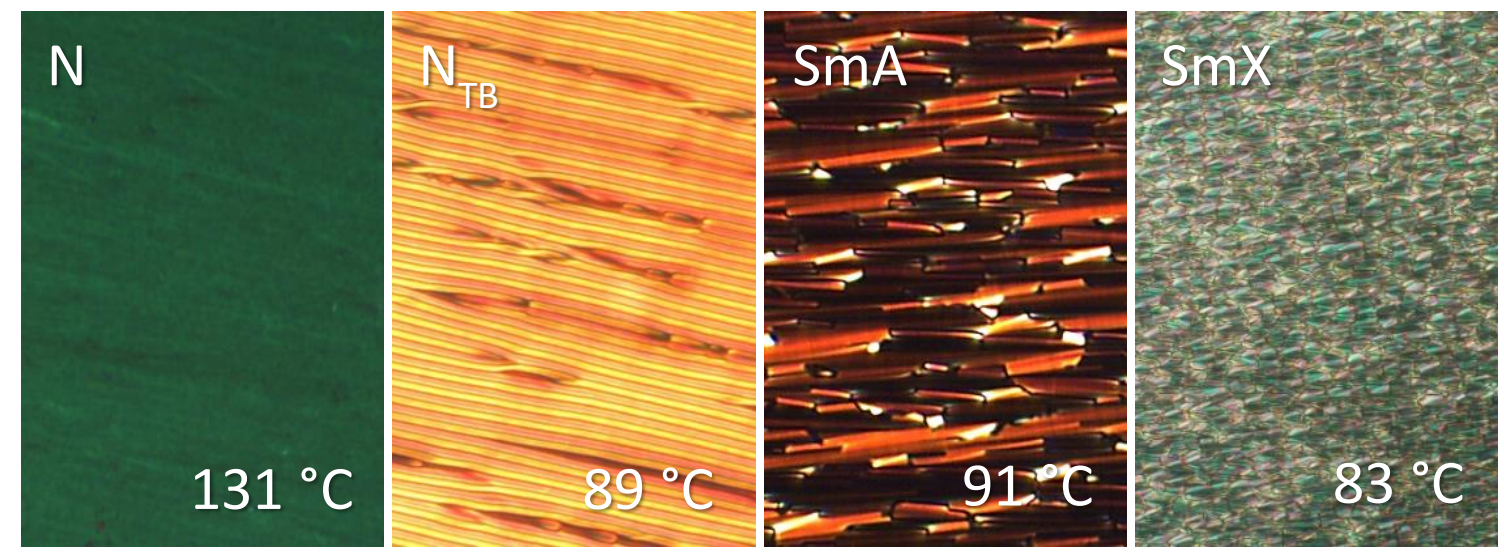

scattering patterns of the complexes with $n<4$ did not allow for the molecular distance along the director to be estimated. For 1OB6OS: $n$ OBA complexes with $n=4$ and 5, the X-ray patterns contained small angle signals corresponding to approximately 1 and $1 / 3$ of the complex's length, indicating an unusual head-to-tail arrangement of the complexes discussed in detail elsewhere. 27. The layer periodicity in the SmA phase for the 1OB6OS:nOBA complexes with $n=7-10$ corresponds to the estimated complex length indicating a monolayer arrangement, see Table 3.

Table 3. Layer thickness (smectic phases) or end-to-end separation between molecules (nematic phases) obtained from integrated X-ray signals for the equimolar 1OB6OS: $n$ OS mixtures. For some mixtures and phases, the observed pattern was too weak to determine the signal position.

\begin{tabular}{ccccc}
\hline $\boldsymbol{n}$ & $\begin{array}{c}\text { Molecular } \\
\text { Length, } \boldsymbol{l} / \AA\end{array}$ & Phase & $\begin{array}{c}\text { Layer Thickness/Longitudinal } \\
\text { Distance, } \boldsymbol{d} / \AA\end{array}$ & $\boldsymbol{d} \boldsymbol{l}$ \\
\hline $\mathbf{4}$ & 37.9 & $\mathrm{~N}$ & $38.6,12.8$ & $1.02,0.34$ \\
\hline \multirow{2}{*}{$\mathbf{5}$} & \multirow{2}{*}{39.4} & $\mathrm{~N}$ & $39.0,13.0$ & $0.99,0.33$ \\
& & $\mathrm{~N}$ & $39.0,13.0$ & $0.99,0.33$ \\
& $\mathrm{SmA}$ & 42.9 & 1.09 \\
\hline $\mathbf{7}$ & 42.5 & $\mathrm{SmA}$ & 43.8 & 1.03 \\
\hline
\end{tabular}




\begin{tabular}{ccccc}
\hline $\mathbf{8}$ & 44.0 & SmA & 45.6 & 1.04 \\
\hline $\mathbf{9}$ & 45.6 & SmA & 47.2 & 1.04 \\
\hline $\mathbf{1 0}$ & 47.1 & SmA & 48.2 & 1.02 \\
\hline
\end{tabular}

Figure 3 shows the dependence of the transition temperatures on the length of the terminal alkyloxy chain, $n$, for the 1OB6OS: $n$ OBA complexes. TNI falls on increasing the terminal chain length reflecting the increased dilution of the interactions between the mesogenic units which promote the formation of the nematic phase. $\mathrm{T}_{\mathrm{TB}} \mathrm{N}$ shows only a weak dependence on $n$ reflecting the predominantly shape driven nature of this transition, and that the spacer largely determines molecular curvature for complexes having similar values of $n$. On increasing the terminal chain length microphase separation is promoted and TSmAN increases rapidly. We will discuss this later together with the behaviour of the CB6OS: $n$ OBA complexes.



Figure 3. Dependence of phase behaviour of the equimolar 1OB6OS: $n$ OBA complexes on the length of the terminal alkyl chain, $n$, where $\times$ represents $\mathrm{T}_{\mathrm{NI}}, \boldsymbol{\mathrm { T }} \mathrm{TmAN}, \boldsymbol{\Delta} \mathrm{T}_{\mathrm{N}_{\mathrm{TB}} \mathrm{N}}$ and $\bullet \mathrm{T}_{\mathrm{T}_{\mathrm{TB}} / \mathrm{SmASmX}}$.

The transitional properties of the equimolar CB6OS: $n$ OBA mixtures are listed in Table 4. CB6OS melts directly into the isotropic phase at $129{ }^{\circ} \mathrm{C}$ and on cooling, crystallisation precludes the observation of liquid crystallinity. Similar to the behaviour seen for the 
1OB6OS: $n$ OBA mixtures (Table 2), CB6OS: $n$ OBA complexes with $n=1-5$ exhibit two nematic phases, an enantiotropic conventional $\mathrm{N}$ phase and a monotropic NTB phase. The $\mathrm{N}$ and Nтв phases were assigned as described earlier: specifically, in planar aligned cells, the $\mathrm{N}$ phase has a uniform texture with a strong degree of flickering and on cooling to the Nтв phase a distinct stripe texture develops and the flickering ceases.

CB6OS: $n$ OBA complexes with $n>5$ do not show the Nтв phase and instead exhibit up to three smectic phases and an enantiotropic conventional $\mathrm{N}$ phase. The smectic phase which appears first on cooling the nematic phase for complexes with $n=6-10$ showed a uniform texture with no optical flickering in planar aligned cells (Figure 4b) and if viewed using untreated glass slides with one surface free, a largely non-birefringent texture was seen (Figure 4f). This phase is therefore assigned as a SmA phase. On further cooling complexes with $n \geq 7$ in untreated slides, a schlieren texture containing two- and four-point brush defects developed from the homeotropically aligned regions, suggesting the formation of a tilted smectic phase (Figure $4 \mathrm{~g})$. Two-brush defects are characteristic of phases in which the mesogenic units are arranged in an anticlinic fashion, suggesting this is the $\mathrm{SmCA}$ phase. In the planar aligned cell, a striped texture is seen (Figure 4c) and such stripes are indicative of a low bend elastic constant 37. This texture resembles that reported for the heliconical smectic phase denoted the SmCтв phase 20,21. However, the emergence of a schlieren texture from homeotropic regions in the preceding SmA phase is not consistent with a heliconical phase in which consecutive layers rotate according to an ideal clock model. In such a system the molecular orientations are averaged over the helix and a homeotropic texture may observed even though the phase is tilted. Recently, however, we have shown that a variant of the heliconical smectic $\mathrm{C}$ phase may be described using a distorted clock model in which the molecular orientations are not averaged over the helix and hence a schlieren texture may be observed. To distinguish between a conventional SmCA phase and a distorted clock smectic Ств phase requires a detailed resonant X-ray diffraction study but the monotropic nature of these phases precludes this possibility. The two lowest temperature smectic phases are strongly monotropic. On cooling from the SmC phase, the schlieren pattern becomes a somewhat smudged, plate-like texture (Figure $4 \mathrm{~h}$ ) and this has been denoted SmX but the texture resembles the that of the SmI phase 36. In the planar aligned cell, the striped texture of the SmC phase is replaced with a mosaic texture containing domains resembling arrowheads pointing in opposite directions (Figure 4d). On cooling the SmX phase, the grey/blue-coloured 'plates' undergo a marked change in birefringence to orange/brown accompanied by the appearance of additional scratch-like defects. This transition tends to be 
simultaneous with crystallisation, and hence was difficult to study. We tentatively assign this as a highly ordered, crystal-type phase such as the $\mathbf{J}$ phase 36 .

Table 4. Transition temperatures and associated entropy changes for the CB6OS: $n$ OBA complexes. All data are extracted from heating DSC traces unless stated otherwise. *Temperature obtained from a DSC cooling trace. $†$ Temperature obtained using POM.

\begin{tabular}{|c|c|c|c|c|c|c|}
\hline$n$ & $\mathrm{Tc}-/{ }^{\circ} \mathrm{C}$ & $\mathrm{TSmX}-{ }^{\circ} \mathrm{C}$ & $\mathrm{T}_{\mathrm{SmCSmA}} /{ }^{\circ} \mathrm{C}$ & $\mathrm{T}_{\text {SmAN }} /{ }^{\circ} \mathrm{C}$ & $\mathrm{T}_{\mathrm{N}_{\mathrm{TB}}} /{ }^{\circ} \mathrm{C}$ & $\mathrm{T}_{\mathrm{NI}} /{ }^{\circ} \mathrm{C}$ \\
\hline 1 & 126 & & & & $110 \dagger$ & 178 \\
\hline 2 & 104 & & & & $106 \dagger$ & 179 \\
\hline 3 & 110 & & & & $102 \dagger$ & 169 \\
\hline 4 & 107 & & & & $95 \dagger$ & 167 \\
\hline 5 & 104 & $72^{*}$ & & & $94 \dagger$ & 161 \\
\hline 6 & 105 & $75^{*}$ & & $86 \dagger$ & & 158 \\
\hline 7 & 103 & $77^{*}$ & $91^{*}$ & $95^{*}$ & & 155 \\
\hline 8 & 122 & $78^{*}$ & $90 \dagger$ & $101 \dagger$ & & 155 \\
\hline 9 & 129 & $76^{*}$ & $99 *$ & 114 & & 152 \\
\hline 10 & 117 & $78^{*}$ & $102^{*}$ & 122 & & 151 \\
\hline$n$ & $\Delta \mathrm{SCr}_{\mathrm{Cr}} / \mathrm{R}$ & $\Delta \mathrm{S} S \mathrm{x} \mathrm{x} / \mathrm{R}$ & $\Delta \mathrm{SSmCSmA}^{\prime} / \mathbf{R}$ & $\Delta \mathrm{SSmAN}_{\mathrm{S}} \mathbf{R}$ & $\Delta \mathrm{S}_{\mathrm{TB}_{\mathrm{B}} \mathrm{N}} / \mathrm{R}$ & $\Delta \mathrm{S}_{\mathrm{NI}} / \mathrm{R}$ \\
\hline 1 & 15.09 & & & & $\approx 0$ & 0.61 \\
\hline 2 & 12.64 & & & & $\approx 0$ & 0.79 \\
\hline 3 & 8.38 & & & & $\approx 0$ & 0.78 \\
\hline 4 & 9.08 & & & & $\approx 0$ & 0.79 \\
\hline 5 & 11.98 & 1.87 & & & $\approx 0$ & 0.66 \\
\hline 6 & 10.17 & 1.19 & & - & & 0.59 \\
\hline 7 & 10.99 & 0.93 & 0.03 & 0.004 & & 0.47 \\
\hline 8 & 13.76 & 0.50 & - & - & & 0.47 \\
\hline 9 & 13.54 & 0.94 & 0.03 & 0.03 & & 0.66 \\
\hline 10 & 11.84 & 0.55 & 0.02 & 0.04 & & 0.59 \\
\hline
\end{tabular}



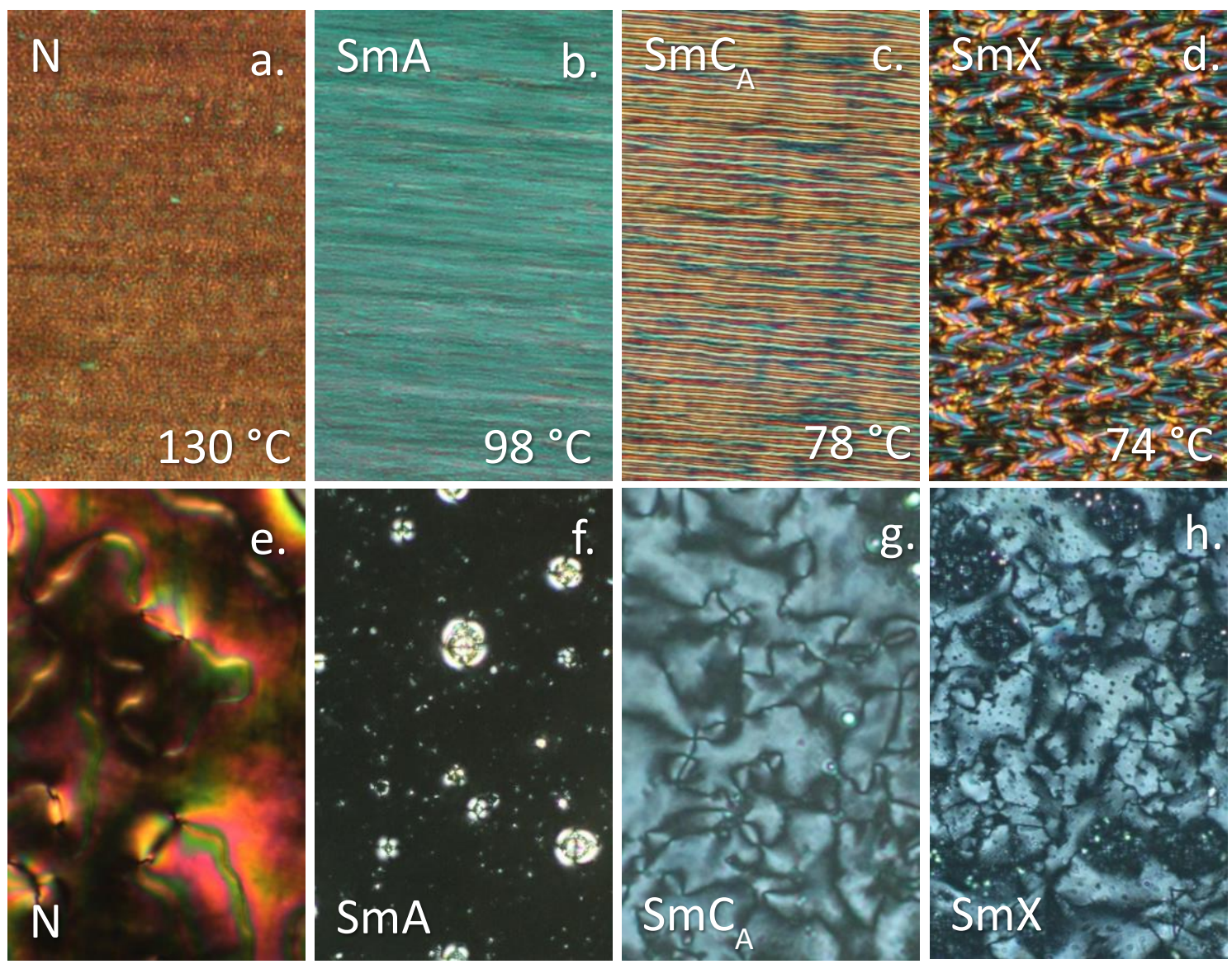

Figure 4. POM textures of CB6OS:9OBA in the $\mathrm{N}, \mathrm{SmA}, \mathrm{SmCA}_{\mathrm{A}}$ and $\mathrm{SmX}$ phases in a planar aligned cell (top) and untreated glass slides (bottom).

The assignments of the nematic phases were confirmed by the observation of broad, diffuse signals in the small- and wide-angle regions in their X-ray diffraction patterns, indicating liquid-like positional ordering of the complexes, see Figure 5. The periodicities in the nematic phases estimated from the position of the broad signal in the small angle region, $d$, are listed in Table 5 along with the calculated molecular length, $l$, for each complex. For the complex with $n=1, d / l \approx 0.5$ suggesting a locally intercalated arrangement of the complexes. On increasing $n$, for the nematic phases of CB6OS:5OBA $d / l \approx 1$ and 0.33 and similar values obtained for 1OB6OS:5OBA have been interpreted in terms of a local head-to-tail arrangement of the complexes 27. For CB6OS:6OBA, $d / l 0.9$ indicating a local monolayer packing. Thus the local structure of the nematic phases changes as $n$ is increased and we will return to this observation later. 
The XRD patterns of the SmA and SmCA phases contain a sharp reflection in the low angle region indicating a lamellar structure, and diffuse wide-angle scattering characteristic of liquidlike ordering within the layers (Figure 5). The values of $d / l$ in these phases suggest monolayer packing, see Table 5. On cooling into the SmX phase, the reflection in the wide-angle region narrows, indicating an increase in the molecular translational ordering within the smectic layer suggesting a hexatic smectic phase; the unambiguous assignment of this would require characterisation of an aligned sample. The position of the small angle reflection remains unchanged (Table 5). The similarities in textures between this phase and that found at low temperatures for the 1OB6OS:5OBA complexes suggest these are the same phases. Crystallisation of the sample precluded the investigation of the lowest temperature smectic phase by X-ray diffraction.


Figure 5. XRD patterns obtained for CB6OS:10OBA in (left to right) the $\mathrm{N}\left(126^{\circ} \mathrm{C}\right), \mathrm{SmA}\left(107^{\circ} \mathrm{C}\right)$, $\mathrm{SmC}_{\mathrm{A}}\left(85^{\circ} \mathrm{C}\right)$ and $\mathrm{SmX}\left(45^{\circ} \mathrm{C}\right)$ phases.

The temperature dependence of the layer spacings, $d$, in the $\mathrm{SmA}, \mathrm{SmC}_{\mathrm{A}}$, and $\mathrm{SmX}$ phases was measured for the $n=10$ complex, see Figure 6. In the SmA phase, the layer spacing increases slightly with decreasing temperature, i.e., a negative temperature layer expansion. At the transition to the SmCA phase $d$ begins to sharply decrease as the molecules tilt. There is no discontinuity in layer spacing at the transition and this is consistent with the weak first-order nature of the transition. The maximum tilt can be estimated from the decrease in the layer thickness using:

$$
\cos \theta=d_{S m C} / d_{S m A}
$$

For the decyl complex $\theta$ was found to be approximately $20^{\circ}$ if we compare the actual layer thickness in the smectic $\mathrm{C}_{\mathrm{A}}$ phase with the value extrapolated from the temperature dependence 
in the SmA phase. A slight increase in layer thickness is observed at the transition to the hexatic SmX phase. The value of $d$ remains lower throughout the SmX phase than in the SmA phase suggesting the $\mathrm{SmX}$ phase is a tilted phase. Cooling the sample into the $\mathrm{Cr}$ phase results in a marked decrease in layer spacing, although it is not clear whether this is the transition to a reversible soft crystal-type phase or crystallisation.

Table 5. Layer thickness (smectic phases) or end-to-end separation between molecules (nematic phases) and lateral spacings obtained from X-ray diffraction patterns of the CB6OS: $n$ OBA series. For some complexes and phases the observed pattern was too weak to determine the signal position.

\begin{tabular}{|c|c|c|c|c|c|}
\hline$n$ & Phase & $\begin{array}{l}\text { Molecular } \\
\text { Length } / l\end{array}$ & $\begin{array}{c}\text { End-to-end separation/Layer } \\
\text { Thickness, } d / \AA\end{array}$ & $\begin{array}{c}\text { Distance Between } \\
\text { Mesogens / } \AA\end{array}$ & $d / l$ \\
\hline 1 & $\overline{N_{T B}}$ & 33.0 & 15.6 & 4.5 & 0.47 \\
\hline \multirow{2}{*}{5} & $\mathrm{~N}$ & \multirow{2}{*}{39.2} & $37.2,12.0$ & 4.5 & $0.95,0.31$ \\
\hline & $\mathrm{N}_{\text {TB }}$ & & $37.1,12.5$ & 4.5 & $0.95,0.32$ \\
\hline 6 & $\mathrm{~N}$ & 40.7 & 36.0 & 4.9 & 0.88 \\
\hline \multirow{2}{*}{7} & $\mathrm{SmC}$ & \multirow{2}{*}{42.2} & 39.9 & 4.3 & 0.95 \\
\hline & SmX & & 42.5 & 4.3 & 1.01 \\
\hline 8 & $\mathrm{SmA}$ & 43.8 & 45.3 & 4.3 & 1.03 \\
\hline \multirow{3}{*}{10} & $\mathrm{SmA}$ & \multirow{3}{*}{45.3} & 46.8 & 4.4 & 1.03 \\
\hline & $\mathrm{SmC}$ & & 45.4 & 4.3 & 1.00 \\
\hline & SmX & & 44.5 & 4.3 & 0.98 \\
\hline
\end{tabular}

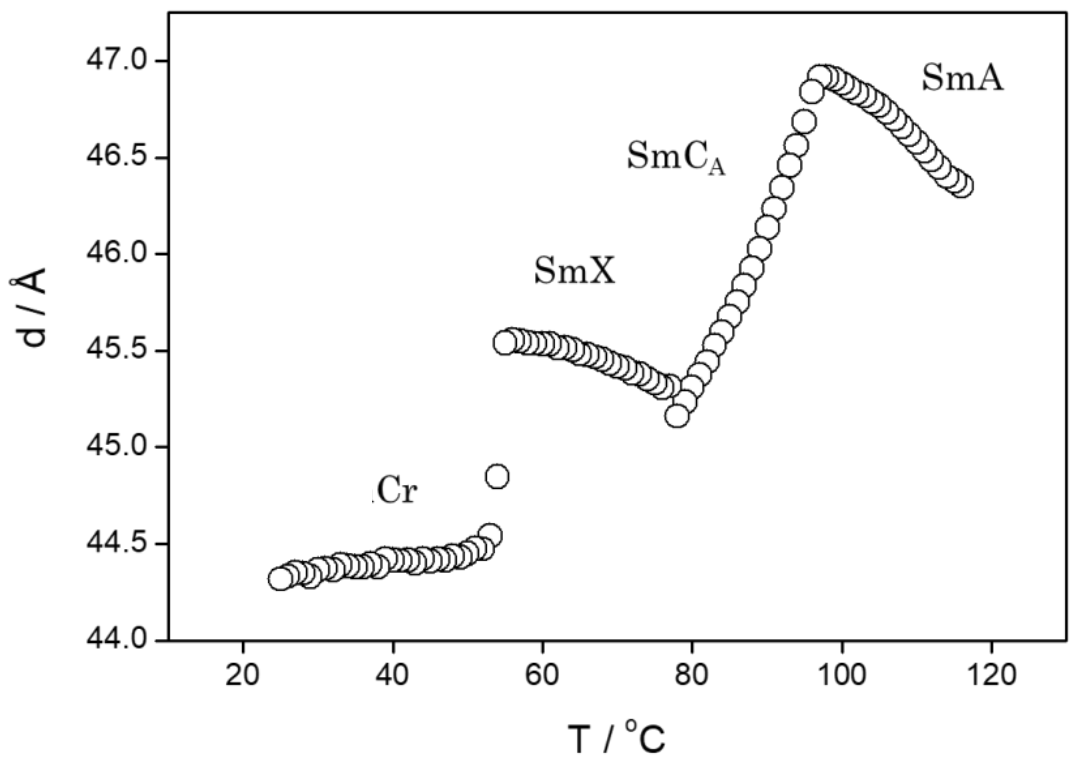

Figure 6. Dependence of the layer spacing on temperature for CB6OS:10OBA. 
The dependence of the transition temperatures on $n$ for the CB6OS: $n$ OBA complexes is shown in Figure 7. The behaviour observed is qualitatively the same as that seen for the 1OB6OS:nOBA complexes in Figure 3. The principal difference is the insertion of the $\mathrm{SmC}_{\mathrm{A}}$ phase for long terminal chain lengths for the CB6OS: $n \mathrm{OBA}$ complexes. It would be tempting to attribute this difference in behaviour to a stabilisation of the anticlinic arrangement of the molecules by antiparallel associations of the cyanobiphenyl fragments. Such a view, however, is not supported by the similar values of $d / l$ observed in the SmA phase for these two sets of materials. It is apparent that both sets of complexes show a progression in terms of the local arrangement of the complexes in the nematic phases but this is not reflected in the transition temperatures which show a regular dependence on $n$. Similar behaviour has been observed for covalently assembled liquid crystal dimers in which the length of the terminal chain is varied 10,11 and thought to suggest that the importance of the local arrangement of the molecules in the nematic phase, specifically their tendency to intercalate, has been overstated in considering the stability of the $\mathrm{N}_{\text {тв }}$ phase. Both sets of complexes show a strong tendency to exhibit smectic phases rather then the $\mathrm{N}_{\text {тв }}$ phase on increasing $n$, and this is seen also for a covalently assembled series of nonsymmetric dimers containing a cyanobiphenyl unit and a three-ring mesogenic moiety 20 . By comparison, non-symmetric dimers consisting of a cyanobiphenyl unit and a two-ring mesogenic moiety have a much weaker tendency to show smectic phases over the same range of terminal chain lengths 10,11 . This behaviour may be attributed to the enhanced interaction strength parameter between the mesogenic units on increasing the length of one. Thus it appears that the hydrogen-bonded complexes behave similarly to their covalently assembled counterparts. 


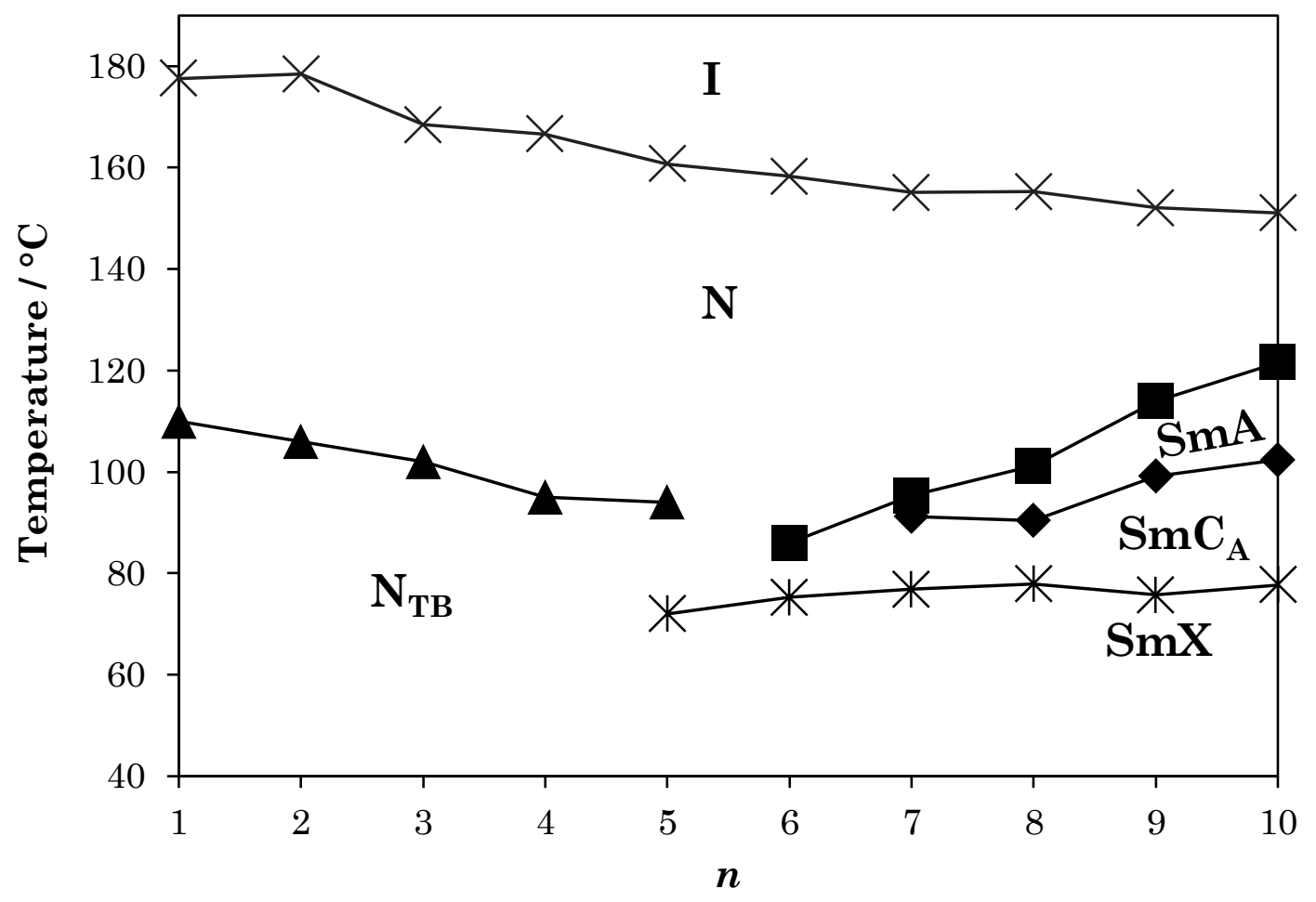

Figure 7. Dependence of phase behaviour of the CB6OS:nOBA on the length of the terminal alkyl

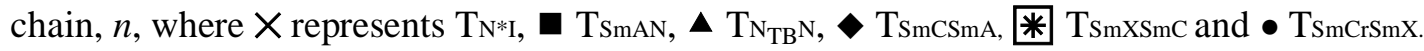

In order to establish to what extent other physical properties of the hydrogen bonded complexes resemble those of covalently assembled dimers, optical birefringence measurements were performed for a selection of materials in the CB6OS: $n$ OBA series. Representative plots of the temperature dependence of the birefringence for those complexes exhibiting nematic to twist-bend nematic phase transitions, $\mathrm{n}=1-5$, and those for longer homologues with a sequence of smectic phases, $n=6-10$, are shown in Figures 8 and 9, respectively. The measured optical birefringence, $\Delta n$, sharply rises at the transition from the isotropic to the nematic phase and on further cooling follows a critical dependence,

$$
\Delta n_{0}=\Delta n_{\max }\left(\frac{T_{N I}-T}{T_{N I}}\right)^{\beta}
$$

where $\mathrm{T}_{\mathrm{NI}}$ is the nematic-isotropic transition temperature and $\Delta \mathrm{n}_{\max }$ is the theoretical birefringence for the material with an ideal orientational order (order parameter, $S=1$ ). 38 The departure of the birefringence from the critical dependence several degrees above the N-NTв transition temperature is attributed to the presence of strong fluctuations of the director in the nematic phase caused by the instantaneous formation of local heliconical states, a pre-transitional effect on approaching the helical twist-bend nematic phase (Figure 8). Such behaviour is consistent with previous reports on the optical birefringence at the N-Nтв transition in covalently bonded liquid crystal dimers 39 . In the Nтв phase, 
we observe a decrease in birefringence which relates to the tilting and averaging of the molecular positions around the helical axis. Furthermore, the birefringence data were also used to determine the conical angle $\Theta_{\text {sat }}$ in the $\mathrm{N}_{\text {тв }}$ phase (inset Figure 8), following a procedure described by Meyer et al. 40 in which the tilt was calculated using,

$$
\theta_{\text {sat }}=\cos ^{-1}\left(\sqrt{\frac{\frac{\Delta n}{\Delta n_{0}} * 2+1}{3}}\right)
$$

An essentially linear increase of the conical angle on decreasing temperature was found below the NNтв phase transition, and in all cases $\Theta$ sat was calculated as approximately $20^{\circ}$.

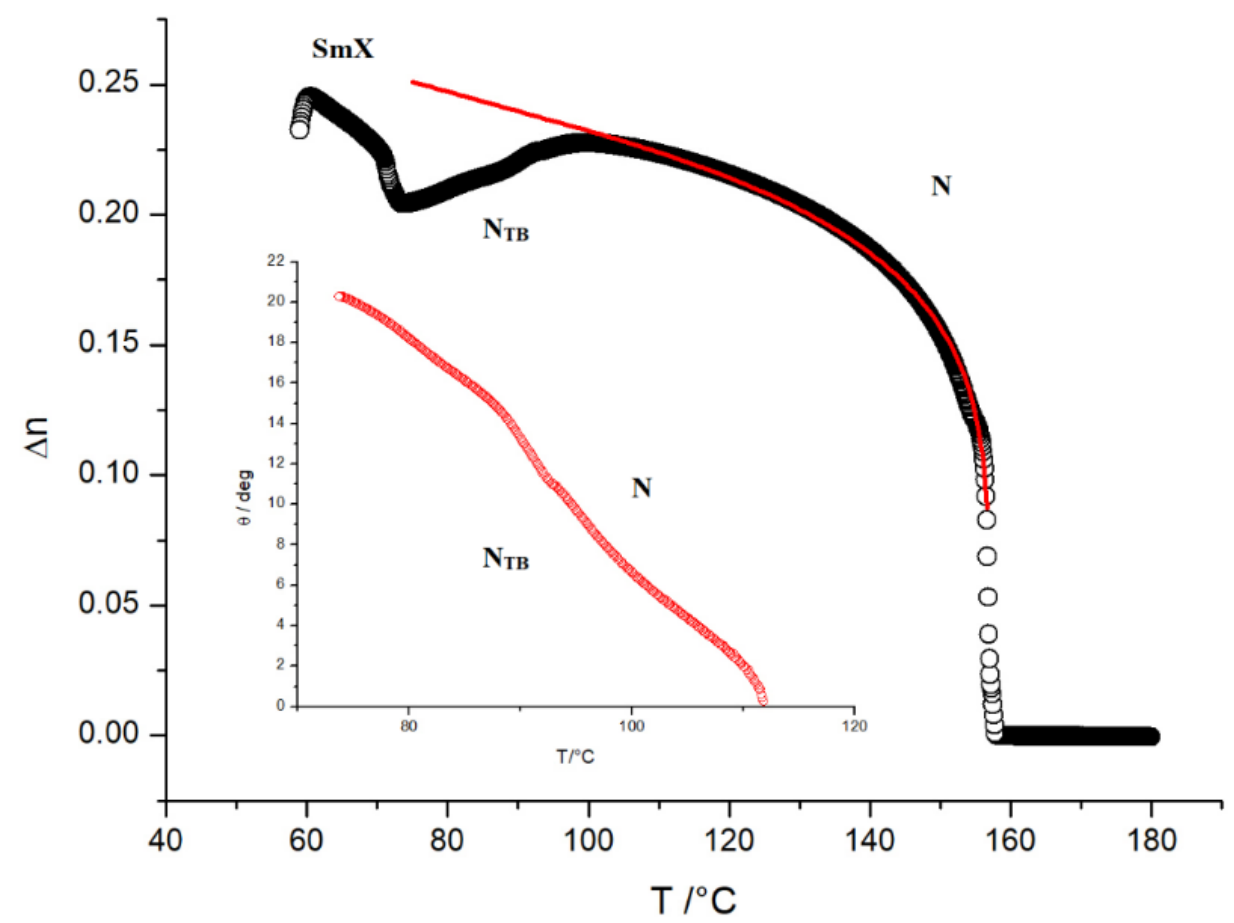

Figure 8. Birefringence vs. temperature for CB6OS:5OBA. The red line is the fitted curve obtained assuming critical temperature dependence. Inset shows a plot of conical angle vs. temperature.

In longer homologues, the SmA-N transition is marked by a small increase in birefringence, due to the increase of orientational order at the onset of layer formation (Figure 9). The subsequent transition to the tilted anticlinic $\mathrm{SmC}_{\mathrm{A}}$ phase lowers the birefringence. The change in tilt angle with temperature in the SmC phase can be fit to the dependence: $\theta=\theta_{S a t}\left(\frac{T_{S m C-S m A-T}}{T_{S m C-S m A}}\right)$ and again, a linear increase of conical angle with decreasing temperature is seen. Values of $\theta$ sat in the $\mathrm{SmC}_{\mathrm{A}}$ phase were 
calculated by the simple estimation: $\cos 2 \theta=\frac{\Delta n_{S m C_{A}}}{\Delta n_{S m A}}$ and were also found to be $\sim 20^{\circ}$. This value is completely in accord with the magnitude of the tilt angle found by $\mathrm{X}$-ray measurements.



Figure 9. Birefringence vs. temperature for CB6OS:10OBA. The red line is the fitted curve obtained assuming critical temperature dependence. Inset shows a plot of conical angle vs. temperature.

\section{Conclusion}

In summary, we report the synthesis, thermal and optical characterisation of two series of supramolecular liquid crystal dimers formed by molecular recognition between unlike H-bond donors and acceptors. The hydrogen bonded complexes of 1OB6OS or CB6OS and $n \mathrm{OBA}(n$ $=1-5$ ) exhibit twist-bend nematic phases providing further examples of the spontaneous formation of chirality in mixtures of achiral compounds. Mixtures of CB6OS and $n \mathrm{OBA}$ with $n=7-10$ may exhibit the heliconical smectic $\mathrm{C}$ phase. In general, the properties of these hydrogen bonded mixtures strongly resemble those of their covalently bonded counterparts. 


\section{References}

1. Tschierske, C. Mirror symmetry breaking in liquids and liquid crystals. Liq. Cryst. 45, 22212252 (2018).

2. Cestari, M. et al. Phase behavior and properties of the liquid-crystal dimer 1",7"-bis(4cyanobiphenyl-4'-yl) heptane: A twist-bend nematic liquid crystal. Phys. Rev. E - Stat. Nonlinear, Soft Matter Phys. 84, 1-20 (2011).

3. Borshch, V. et al. Nematic twist-bend phase with nanoscale modulation of molecular orientation. Nat. Commun. 4, 1-8 (2013).

4. Walker, R., Storey, J. M. D., Imrie, C. T., Pociecha, D. \& Gorecka, E. The Chiral Twist-Bend Nematic Phase (N*TB). Chem. - A Eur. J. (2019). doi:10.1002/chem.201903014

5. Meyer, R. B. Structural problems in liquid crystal physics. in Les Houches Summer School in Theoretical Physics, Molecular Fluids 316-320 (Gordon and Breach, 1976).

6. Dozov, I. On the spontaneous symmetry breaking in the mesophases of achiral banana-shaped molecules. Europhys. Lett. 56, 247-253 (2001).

7. Paterson, D. A. et al. Understanding the twist-bend nematic phase: The characterisation of 1(4-cyanobiphenyl-4'-yloxy)-6-(4-cyanobiphenyl-4'-yl)hexane (CB6OCB) and comparison with CB7CB. Soft Matter 12, 6827-6840 (2016).

8. Mandle, R. J. Designing Liquid-Crystalline Oligomers to Exhibit Twist-Bend Modulated Nematic Phases. Chem. Rec. 18, 1341-1349 (2018).

9. Pocock, E. E., Mandle, R. J. \& Goodby, J. W. Molecular shape as a means to control the incidence of the nanostructured twist bend phase. Soft Matter 14, 2508-2514 (2018).

10. Paterson, D. A. et al. The role of a terminal chain in promoting the twist-bend nematic phase: the synthesis and characterisation of the 1-(4-cyanobiphenyl-4'-yl)-6-(4alkyloxyanilinebenzylidene-4'-oxy)hexanes. Liq. Cryst. 45, 2341-2351 (2018).

11. Walker, R. et al. Molecular curvature, specific intermolecular interactions and the twist-bend nematic phase: the synthesis and characterisation of the 1-(4-cyanobiphenyl-4'-yl)-6-(4alkylanilinebenzylidene-4'-oxy)hexanes (CB6O.m). Soft Matter 15, 3188-3197 (2019).

12. Knežević, A. et al. Induced smectic phase in binary mixtures of twist-bend nematogens. Beilstein J. Nanotechnol. 9, 1297-1307 (2018).

13. Imrie, C. T., Henderson, P. A. \& Yeap, G. Y. Liquid crystal oligomers: Going beyond dimers. Liq. Cryst. 36, 755-777 (2009).

14. Cruickshank, E. et al. Sulfur-linked cyanobiphenyl-based liquid crystal dimers and the twistbend nematic phase. Liq. Cryst. 46, 1595-1609 (2019).

15. Imrie, C. T. \& Henderson, P. A. Liquid crystal dimers and higher oligomers: Between monomers and polymers. Chem. Soc. Rev. 36, 2096-2124 (2007).

16. Xiang, J. et al. Electrically tunable laser based on oblique heliconical cholesteric liquid crystal. Proc. Natl. Acad. Sci. U. S. A. 113, 12925-12928 (2016).

17. Xiang, J. et al. Electrically tunable selective reflection of light from ultraviolet to visible and infrared by heliconical cholesterics. Adv. Mater. 27, 3014-3018 (2015).

18. Dawood, A. A. et al. On the twist-bend nematic phase formed directly from the isotropic phase. Liq. Cryst. 43, 2-12 (2016). 
19. Archbold, C. T., Davis, E. J., Mandle, R. J., Cowling, S. J. \& Goodby, J. W. Chiral dopants and the twist-bend nematic phase-induction of novel mesomorphic behaviour in an apolar bimesogen. Soft Matter 11, 7547-7557 (2015).

20. Abberley, J. P. et al. Addendum: Heliconical smectic phases formed by achiral molecules. Nat. Commun. 9, 2856 (2018).

21. Salamończyk, M. et al. Multi-level chirality in liquid crystals formed by achiral molecules. Nat. Commun. 10, (2019).

22. Sreenilayam, S. P., Panov, V. P., Vij, J. K. \& Shanker, G. The NTB phase in an achiral asymmetrical bent-core liquid crystal terminated with symmetric alkyl chains. Liq. Cryst. 44, 244-253 (2017).

23. Tuchband, M. R. et al. Distinct differences in the nanoscale behaviors of the twist-bend liquid crystal phase of a flexible linear trimer and homologous dimer. Proc. Natl. Acad. Sci. U. S. A. 166, 10698-10704 (2019).

24. Mandle, R. J. \& Goodby, J. W. A Nanohelicoidal Nematic Liquid Crystal Formed by a NonLinear Duplexed Hexamer. Angew. Chemie - Int. Ed. 57, 7096-7100 (2018).

25. Mandle, R. J., Stevens, M. P. \& Goodby, J. W. Developments in liquid-crystalline dimers and oligomers. Liq. Cryst. 44, 2046-2059 (2017).

26. Jansze, S. M., Martínez-Felipe, A., Storey, J. M. D., Marcelis, A. T. M. \& Imrie, C. T. A twistbend nematic phase driven by hydrogen bonding. Angew. Chemie - Int. Ed. 54, 643-646 (2015).

27. Walker, R. et al. Spontaneous chirality through mixing achiral components: A twist-bend nematic phase driven by hydrogen-bonding between unlike components. Chem. Commun. 54, 3383-3386 (2018).

28. Paterson, D. A. et al. Azobenzene-based liquid crystal dimers and the twist-bend nematic phase. Liq. Cryst. 44, 2060-2078 (2017).

29. Abberley, J. P., Storey, J. M. D. \& Imrie, C. T. Structure-property relationships in azobenzenebased twist-bend nematogens. Liq. Cryst. 00, 1-13 (2019).

30. Paterson, D. A., Abberley, J. P., Harrison, W. T. A., Storey, J. \& Imrie, C. T. Cyanobiphenylbased liquid crystal dimers and the twist-bend nematic phase. Liq. Cryst. 44, 127-146 (2017).

31. Kato, T. et al. Hydrogen-Bonded Liquid Crystals. Novel Mesogens Incorporating Nonmesogenic Bipyridyl Compounds through Complexation between H-Bond Donor and Acceptor Moieties. Chem. Mater. 5, 1094-1100 (1993).

32. Kato, T. \& Fréchet, J. M. J. New Approach to Mesophase Stabilization through HydrogenBonding Molecular Interactions in Binary Mixtures. J. Am. Chem. Soc. 111, 8533-8534 (1989).

33. Martínez-Felipe, A. \& Imrie, C. T. The role of hydrogen bonding in the phase behaviour of supramolecular liquid crystal dimers. J. Mol. Struct. 1100, 429-437 (2015).

34. Jensen, J., Grundy, S. C., Bretz, S. L. \& Hartley, C. S. Synthesis and characterization of selfassembled liquid crystals: P-alkoxybenzoic acids. J. Chem. Educ. 88, 1133-1136 (2011).

35. El Sayed, S. et al. 2,4-Dinitrophenyl Ether-Containing Chemodosimeters for the Selective and Sensitive in Vitro and in Vivo Detection of Hydrogen Sulfide. Supramolecular Chemistry 27, 244-254 (2015).

36. Attard, G. S. et al. Non-symmetric dimeric liquid crystals The preparation and properties of the $\alpha$-(4-cyanobiphenyl-4'-yloxy)- $\omega$-(4-n-alkylanilinebenzylidene-4'-oxy)alkanes. Liq. Cryst. 
16, 529-581 (1994).

37. Salili, S. M. et al. Spontaneously modulated chiral nematic structures of flexible bent-core liquid crystal dimers. Liq. Cryst. 44, 160-167 (2017).

38. Haller, I. Thermodynamic and static properties of liquid crystals. Prog. Solid State Chem. 10, 103-118 (1975).

39. Pociecha, D. et al. Critical behavior of the optical birefringence at the nematic to twist-bend nematic phase transition. Phys. Rev. E 98, 1-5 (2018).

40. Meyer, C., Luckhurst, G. R. \& Dozov, I. The temperature dependence of the heliconical tilt angle in the twist-bend nematic phase of the odd dimer CB7CB. J. Mater. Chem. C 3, 318-328 (2015). 